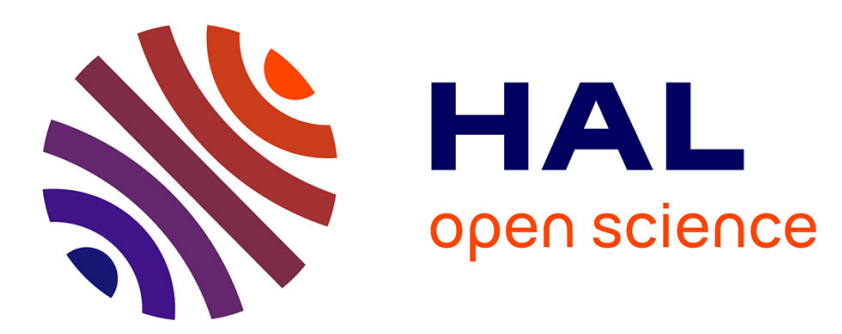

\title{
Influence of a Functional Core Stability Program on Trunk and Knee Joint Biomechanics in Female Athletes During Lateral Movements
}

Guillaume Mornieux, Elmar Weltin, Craig Friedman, Monika Pauls, Shad Forsythe, Albert Gollhofer

\section{To cite this version:}

Guillaume Mornieux, Elmar Weltin, Craig Friedman, Monika Pauls, Shad Forsythe, et al.. Influence of a Functional Core Stability Program on Trunk and Knee Joint Biomechanics in Female Athletes During Lateral Movements. Journal of Strength and Conditioning Research, In press, 10.1519/JSC.0000000000003212 . hal-03102220

\section{HAL Id: hal-03102220 \\ https://hal.univ-lorraine.fr/hal-03102220}

Submitted on 23 Apr 2021

HAL is a multi-disciplinary open access archive for the deposit and dissemination of scientific research documents, whether they are published or not. The documents may come from teaching and research institutions in France or abroad, or from public or private research centers.
L'archive ouverte pluridisciplinaire HAL, est destinée au dépôt et à la diffusion de documents scientifiques de niveau recherche, publiés ou non, émanant des établissements d'enseignement et de recherche français ou étrangers, des laboratoires publics ou privés. 
Title: Influence of a functional core stability program on trunk and knee joint biomechanics in female athletes during lateral movements.

Running Head: Core stability training program and lateral movements.

Laboratory where the research was conducted: Institute of Sport and Sport Science, Freiburg, Germany

Mornieux Guillaume ${ }^{1}$, Weltin Elmar ${ }^{2}$, Friedman Craig ${ }^{3}$, Pauls Monika ${ }^{4}$, Forsythe Shad $^{5}$ and Gollhofer Albert ${ }^{2}$

${ }^{1}$ Faculty of Sport Sciences, DevAH, University of Lorraine, Nancy, France

${ }^{2}$ Institute of Sport and Sport Science, University of Freiburg, Freiburg, Germany

${ }^{3}$ Exos, Performance Innovation Team, Phoenix, AZ, Unites Stated

${ }^{4}$ Institute of Sport Science, Technische Universität Kaiserslautern, Kaiserslautern, Germany

${ }^{5}$ Arsenal Football Club, London, United Kingdom

Corresponding Author: Guillaume Mornieux

Faculty of Sport Sciences, 30 rue du Jardin Botanique - 54600 Villers-lès-Nancy, France Tel.: +33-3-72746739 / Fax: +33-3-72746712

Email: guillaume.mornieux@univ-lorraine.fr 

in female athletes during lateral movements 


\begin{abstract}
Trunk positioning has been shown to be associated with knee joint loading during athletic tasks, especially changes of direction. Quasi-static and conventional core stability (CS) training did not improve the biomechanics of cutting maneuvers according to reports in the literature. The purpose of the present study was to test whether a 5-week dynamic and functional CS training program would improve trunk positioning and knee joint control during lateral movements.

Nineteen female athletes participated in a longitudinal study, where lateral reactive jumps (LRJ) and unanticipated cuttings were performed pre and post CS training (15 training sessions within 5 weeks) while 3D kinematics and kinetics were measured. Additionally, core endurance and power were evaluated.

CS training increased trunk rotation in the new movement direction $(\mathrm{p}=0.02)$ during LRJ. However, lateral trunk lean was significantly increased during LRJ (from $5.6^{\circ}$ to $7^{\circ}$ ) and cuttings (from $3.8^{\circ}$ to $5.8^{\circ}$ ) after CS training. Knee joint abduction moment was not influenced. Finally, core endurance $(\mathrm{p}<0.01)$ and core power $(\mathrm{p}<0.001)$ were significantly improved after CS training.

A 5-week dynamic and functional core stability training program improved core muscles endurance and power. This might explain the better trunk rotation towards the new movement direction during LRJ, however, it was not effective during unanticipated cuttings.

The practical application of this research will allow strength and conditioning professionals to setup training programs with simple and relevant exercises to increase the core endurance and power of trained individuals.
\end{abstract}

Keywords: cuttings, perturbation, trunk lateral lean, knee abduction moment, agility. 


\section{INTRODUCTION}

Epidemiological studies have demonstrated that female athletes have a relative higher risk of sustaining non-contact anterior cruciate ligament (ACL) injuries than male athletes during sports such as soccer or basketball $(1,30)$. As a countermeasure, preventive neuromuscular training (PNMT) interventions have been designed to reduce knee joint injuries, especially ACL ruptures in female athletes $(25,32)$. It has been generally accepted that PNMT significantly reduces ACL injuries, especially when emphasis is put on specific training exercises based on strengthening and trunk neuromuscular control (32).

During athletic movements, trunk neuromuscular control is of interest since it influences trunk load and motion and therefore knee joint loads $(12,16)$. Hewett and Myer (12) proposed a knee injury model that accounted for both the trunk and hip joints, since increased trunk lateral lean is reportedly associated with higher external knee abduction moments. Moreover, a greater lateral trunk position has been shown during anterior cruciate ligament injury situations in female athletes (14). In a prospective study over 3 years, Zazulak et al. (39) demonstrated greater trunk displacement after a sudden force release in athletes who subsequently sustained a knee injury. The association between trunk positioning and knee joint loads has been investigated during changes of direction or cutting maneuvers, since these complex dynamic tasks represent one of the most common playing situations that could lead to a non-contact ACL injury $(2,20)$. During cutting maneuvers, increased trunk lateral lean has been demonstrated to be positively associated with increased knee abduction moments $(8,18,36)$. Furthermore, increased knee joint loading has been associated with increased trunk rotation away from the stance limb $(8,11)$. The redirection of the center of mass towards the new movement direction seems to be a key factor, as demonstrated by Donnelly et al. (10), when producing an optimized whole-body kinematics solution to reduce the knee joint 
abduction moment. This is also in agreement with David et al. (6), who described a cutting movement strategy with forefoot striking in combination with pre-orientation of the upper body towards the new movement direction to reduce knee joint loads. Accordingly, any improvement in trunk and pelvis positioning during cutting maneuvers is of interest with regard to knee joint loading. Thus, core stability $(15,19)$ should be additionally targeted in existing PNMT interventions aimed at modifying cutting maneuver biomechanics (26).

Although literature has reported that quasi-static core stability (CS) training could significantly reduce the vertical ground reaction force (3) and change leg kinematics and joint moments (28) during drop jump landings, such biomechanical improvements were not found after quasi-static CS training during cutting maneuvers (17). In this latter study, 6 weeks of CS training did not improve core strength, trunk control or knee joint loading during cutting maneuvers relative to resistance training. Moreover, a 6-week dynamic CS program (38) did not influence trunk or pelvis kinematics or knee moments during unanticipated cuttings. In addition, a 4-week training program combining lateral movements and perturbation influenced trunk and pelvis rotation in the same manner as a standard plyometric training, and no influence was found on knee joint moments during unanticipated cuttings (35). Therefore, enhanced dynamic and functional CS training is needed to effectively improve trunk and pelvis control towards the new movement direction during unanticipated cutting maneuvers. However, knee joint loads, and especially knee joint abduction moment, as a relevant parameter associated with knee injury (13), seems not to be influenced by such training, although such training should be beneficial for athletic performance. Indeed, it has been reported in the literature that the endurance $(17,33)$ and strength $(29,33)$ of core muscles was improved and specific athletic performance parameters were increased $(29,33,37)$ after CS programs. 
The purpose of the present study was to evaluate the influence of a 5-week dynamic and functional CS training program on trunk and pelvis control and knee joint control during dynamic lateral sporting movements, as well as athletic performance. We tested the hypotheses that i) trunk and pelvis kinematics would be better orientated towards the new movement direction after CS training; ii) the knee joint abduction moment would remain at the same level during cuttings after CS training; and iii) jump performance, agility, core endurance and power would be enhanced following CS training.

\section{METHODS}

\section{Experimental Approach to the Problem}

This study proposed a training intervention over 5 weeks to determine the effects of a dynamic and functional core stability program on cutting biomechanics and athletic performance. Female participants were recruited according to their relative higher risk of sustaining non-contact ACL injuries (1). Although no control group was used in the present study, it was assumed that the results provided by Weltin et al. (35) would constitute reference data to interpret the influence of CS training. The rationale for this was the relatively similar training volume used in their study (three times per week over four weeks) for two relevant training programs, i.e., a modified plyometric training program originally designed by Chimera et al. (4) and a plyometric training program with perturbation called PPT training in the Weltin' study (35), in addition to their comparable testing procedure. Indeed, the methodology used to assess biomechanics, the perturbed lateral reactive jump and cutting maneuver conditions, and the dependent biomechanical variables were exactly the same in this latter study as in the present one.

Pre and post training measurements evaluated three-dimensional trunk and pelvis kinematics during perturbed lateral reactive jumps, and additionally the knee joint moment during $45^{\circ}$ 
cutting maneuvers. Core endurance and power as well as jump performance and agility were also evaluated.

\section{Subjects}

An a priori power analysis was conducted to estimate the sample size based on the measured improvement in the intervention group (effect size of 0.6 for trunk kinematic data) of recent published research (35). It was estimated that 19 participants were needed to achieve $80 \%$ statistical power with an alpha level of 0.05 . This sample size is in line with other studies (17). Twenty-two females were initially enrolled in the study to account for possible dropouts. All participants were interviewed individually to assess eligibility for participation. They had to meet the following inclusion criteria: at least five years of active participation in team sports with high exposure to running, jumping and cutting tasks, no additional core stability or balance training, no history of anterior cruciate ligament injury, trunk or back injury, neurological disorders or any current pain. Participants trained at least three times a week (up to five times) and played at different levels ( $1^{\text {st }}$ to $5^{\text {th }}$ division). Three participants withdrew from the study due to injuries (two ankle ligament injuries and one hamstring muscle tear) that occurred during their regularly scheduled season practices and matches, resulting in more than two missed training sessions in the present intervention. Accordingly, 19 female athletes were included in the analysis $(22 \pm 3$ years, $172 \pm 7 \mathrm{~cm}, 61 \pm 7 \mathrm{~kg}, 9 \pm 4$ years sport experience).

The study was approved by the local ethics committee (approval number: 390/13) and conformed to the requirements stipulated in the Declaration of Helsinki. All participants were informed about the procedures, purpose, benefits and possible risks associated with the experimental set-up prior to signing an institutionally approved informed consent document to participate in the study. 


\section{Procedures}

Intervention. All participants took part in regularly scheduled season practices and matches, in addition to attending the present core stability (CS) program three times per week for five weeks (15 sessions). The present CS training (Table 1) involved 15 training sessions. Each session lasted for 30min and was based on warm-up exercises, medicine ball throws, a cable lift task, and different variations of lateral shuffles. These specific exercises are relevant with respect to cutting maneuver biomechanics, since i) they are dynamic, ii) they target the core but also involve the whole-body, and iii) they are functional with respect to the nature of a cutting maneuver, involving trunk and pelvis segment rotations while pushing medially and upwards with the stance leg into the new movement direction. Together with a progressive increase in the intensity and the introduction of perturbation in the latter sessions, the present CS training should be more effective than previously tested programs seeking to improve cutting maneuver biomechanics $(17,35,38)$. Participants were given instructions and illustrations about the exercises and were supervised by athletic coaches to ensure proper and effective execution of the movement techniques. Particular attention was paid to the rotation of the hip during the medicine ball throw. Indeed, hips should lead the movement followed by the upper body. The ball should be held at the hip level opposite the wall, and the foot should rotate together with the hip. Focus was set on turning the shoulders and torso, as well as combining familiar movements of upright rows and an incline press during the cable lift. During shuffles, athletes had to keep their weight forward to maintain an upright torso position with respect to the vertical axis in the frontal plane and to point their toes forwards. The motor learning principles behind the training procedure referred mainly to the internal focus of attention and explicit learning during the first 6 training sessions. Then, an external focus of attention and implicit learning were introduced by focusing on the medicine ball 
("trying to launch the ball from the hip") or performing shuffles using mirror drills with partners. Moreover, differential learning was initiated by having a variety of movement patterns targeting the trunk and pelvis segments re-orientation throughout the intervention and changing the environment (different training partners, resistances, and perturbations).

Table 1 about here

Testing. Participants performed different lateral movements and performance tests prior to CS training (PRE) and after the CS training intervention (POST). Post testing was scheduled in order to match conditions of the PRE measurements, in terms of day of the week, time of the day, and sleep.

Firstly, participants performed perturbed lateral reactive jumps (LRJ) to focus on movement control during a functional task associated with high trunk lateral lean in the frontal plane. From a standing position, subjects performed a lateral reactive movement by jumping to their left side and then pushing off with their left leg to get back to their original position. In order to induce lateral perturbations, subjects performed LRJ on a motor-driven plate, which could move in the horizontal (medio-lateral) direction. The specially designed motor-driven plate (University of Sport Science, Freiburg, Germany) was comprised of two independently moving plates $(1.15 \mathrm{~m} \times 0.3 \mathrm{~m})$, each driven by a separate motor (AKM51G, Danaher Motion, Düsseldorf, Germany). LRJ were performed either without any perturbation (stable condition) or with a perturbation to the left (sliding condition) or to the right (counteracting condition) as soon as the foot made initial contact with the plate. Each perturbation consisted of a $10 \mathrm{~cm}$ translation. Unpredictability was introduced using these three different landing modalities (stable, sliding, and counteracting) that were unknown prior to movement execution. The perturbation was triggered by breaking a light beam (M18 series, Panasonic 
Electric Works Europe AG, Holzkirchen, Germany). Details of this material have been previously described in the literature (23).

Secondly, participants were asked to perform either cutting maneuvers to $45^{\circ}$, crossovers to $20^{\circ}$, or straight runs on a force plate (BP600900, AMTI®, Watertown, USA) after approaching the force plate (FP) at $4 \pm 0.2 \mathrm{~m} . \mathrm{s}^{-1}$. The direction of the movement was indicated in a randomized order by a light signal occurring $650 \mathrm{~ms}$ before the left foot contacted the FP. The visual light stimulus was triggered automatically during the approach run via a light switch (M18 series, Panasonic Electric Works Europe AG, Holzkirchen, Germany). The 45 cutting maneuver to the right was the only movement further analyzed due to its relevance when investigating performance and injury aspects of changing direction tasks. Details of these procedures have been previously described in the literature (24). The test-retest reliability of these specific biomechanical evaluations were additionally tested for nine participants providing an intraclass correlation coefficient (ICC) value for the different dependant variables. The reliability of the trunk, pelvis, and knee kinematics during LRJ was good, with ICC values respectively above $0.94,0.89$ and 0.87 when averaged over the three conditions. During cuttings, the reliability of the trunk and pelvis kinematics was good (ICC > 0.81 and 0.85 , respectively), and the ICC values for knee joint moments were 0.88 (flexion), 0.72 (abduction), and 0.52 (external rotation). Based on the ICC values, the standard error of measurement (SEM) was determined to provide further valuable information in order to interpret significant effects detected during LRJ and cuttings with the present repeated measures design.

Finally, core endurance and power, jump performance, and agility were evaluated. Holding times during a prone plank and a left side plank were used to evaluate core endurance. Time was stopped when participants could not hold the plank anymore. Jump performance was evaluated by broad and lateral jumps, agility with the 5-10-5 yards test and core power with a 
cable lift test. This latter test targets the action of the hips, torso rotators, upper back, chest and shoulders while turning the trunk away from the strength machine as the hands first pull the rope cable handle towards the chest and then push up and away (Figure 1).

Figure 1 about here

The rationale for all these tests was the importance of agility, leg muscle strength, core endurance and power for high performance in team ball sports. The best out of two broad jumps and two lateral jumps to the right were determined from the distance measured. The time taken to accomplish the agility test was measured manually. The cable lift test was conducted three times at $70 \%$ of the one-repetition maximum. The vertical acceleration signal of an accelerometer (University of Sport Science, Freiburg, Germany) placed on the top of the weight stack was filtered with a low pass Butterworth filter $\left(4^{\text {th }}\right.$ order, $20 \mathrm{~Hz}$ cut-off frequency) and then integrated to obtain velocity. Lift force was calculated based on Newton's second law. Finally, core power was determined as the product of force and velocity. The good reliability for lower back stabilization exercises (ICC > 0.96), as well as for the 5-10-5 yards agility test (ICC > 0.87) have been reported in the literature $(21,31)$. The test-retest reliability of this specific cable lift task across the nine participants was additionnally evaluated $(\mathrm{ICC}=0.67)$.

All participants were familiar with such movements due to their sport practice and were given enough practice trials to feel comfortable with all task modalities prior to the measurements.

Data analysis. Three-dimensional leg kinematics were recorded using reflective skin markers ( $\varnothing 14 \mathrm{~mm}$ ) placed on anatomical landmarks of the subjects' trunk (three markers), pelvis (four markers), left thigh (three markers), shank (four markers), and foot (six markers). The 
markers were captured with a 12-camera motion analysis system (Vicon $\mathrm{MX}^{\mathrm{TM}}$, VICON Motion Systems Ltd. ${ }^{\circledR}$, Oxford, UK) with a sampling frequency of $200 \mathrm{~Hz}$. A static trial with the subject standing in a pre-defined neutral position was used to define the kinematic model and to calculate segment lengths and joint centers. The kinematic modeling was performed using the motion analysis software (Vicon Nexus 1.6.1, VICON Motion Systems Ltd.®, Oxford, UK). Joint kinematics in three rotational degrees of freedom were determined using an yxz Euler rotation sequence of the respective segment coordinate system according to the global reference system defined in Vicon. This rotation sequence was around the flexion/extension axis first, then around the abduction/adduction axis, followed by rotation around the external/internal axis. This marker placement and kinematic modeling were used in previously published articles $(22,36)$.

The marker trajectories and ground reaction force signals were both filtered with a low pass Butterworth filter ( $4^{\text {th }}$ order, $15 \mathrm{~Hz}$ cut-off frequency) prior to calculating external joint moments with a standard inverse dynamics approach. Three-dimensional kinematic data of the trunk and pelvic segments were analyzed to understand the influence of CS training on core control. The three-dimensional kinematics and kinetics of the knee joint were further analyzed to determine the influence of CS training on knee joint stability and loading. Both kinematics and kinetics were measured during cutting maneuvers, but only kinematics was observed during LRJ. These variables were analyzed at the time of their peak values during the first half of ground contact during LRJ to give some insight into how CS training influences the range of motion of the different body segments. However, during cutting maneuvers, the different variables were determined at the time of peak knee abduction moment. The trunk and pelvis kinematics were defined relative to the global coordinate system, i.e., deviations from the vertical axis for trunk forward and lateral leans, and for pelvis forward and medial tilts, and from the anterior-posterior axis for trunk and pelvis right 
axial rotation. These parameters were positive for an orientation in the new movement direction (i.e., a medial lean and rotation in the right direction). Knee rotation parameters were positive for an external rotation.

\section{Statistical Analyses}

The selected parameters were averaged across eight trials for the different LRJ modalities and the $45^{\circ}$ cutting maneuver, whereas time for the 5-10-5 yards test was reported and the highest $\mathrm{CMJ}$ and mean peak power value over three cable lift trials were calculated. These parameters served as the basis for the statistical analysis (Statistica 12, StatSoft, Inc. ${ }^{\text {, }}$, Tulsa, OK, US). All results are presented as group mean \pm standard deviation (SD). After having confirmed that the data followed a normal distribution (Shapiro-Wilk W), sphericity was verified by means of a Mauchly's test. The influence of CS training on dependent variables for LRJ modalities was calculated using a two-way analysis of variance (General Linear Model) with repeated measures to test for the main effects of and interactions between CS training (PRE vs. POST) and LRJ modality (stable vs. sliding vs. counteracting). If a statistically significant LRJ modality main effect was observed, Bonferroni corrected paired comparisons determined where the differences occurred. The influence of CS training (PRE vs. POST) on the dependent variables for the cutting maneuver and performance tasks was analyzed using paired two-tailed t-tests. The magnitude of the changes was assessed with effect size (ES) and a 95\% confidence interval (CI). The ES was evaluated by means of Cohen's d values for repeated measures designs. The ES was considered trivial (0-0.19), small (0.20-0.49), medium (0.50-0.79), or large (>0.80) according to Cohen (5). The level of significance was set at $p<$ 0.05 .

\section{RESULTS}


There was a significant main effect of CS training on trunk lateral lean $(p=0.04)$, trunk axial rotation $(p=0.002)$, pelvis medial tilt $(p=0.002)$, and pelvis axial rotation $(p<0.001)$ during LRJ, but no significant influence at the knee joint level (Table 2). SEM values for trunk lateral lean and rotation were $0.7^{\circ}$ and $1.4^{\circ}$, respectively, while the pelvis medial tilt and rotation had SEM values of $1.3^{\circ}$ and $1.7^{\circ}$, respectively.

Table 2 about here

Statistical analysis revealed a significant main effect of the LRJ modality on trunk forward lean and trunk lateral lean (both $p<0.001)$, pelvis medial tilt $(p<0.001)$, knee flexion, abduction, and rotation ( $p<0.001, p=0.04$ and $p<0.001$, respectively). Significant interaction effects (CS training $\times$ LRJ modality) were found only for trunk axial rotation $(p=$ $0.01)$ and knee joint abduction $(p=0.03)$. Specifically, pairwise comparisons revealed that stable, sliding, and counteracting modalities yielded significantly different values from each other for trunk forward lean $(p<0.007$ for all), trunk lateral lean $(p<0.001$ for all), pelvis medial tilt $(p<0.002$ for all), knee flexion $(p<0.001$ for all), knee abduction $(p<0.05$ for all), and knee rotation $(p<0.001$ for all).

During the cutting maneuver (Table 3), trunk forward lean and axial rotation were not influenced after CS training, whereas trunk lateral lean was significantly increased $(p=0.03)$. Pelvis kinematics was not significantly influenced by CS training. Knee joint flexion moment decreased significantly $(p=0.01)$ after CS training, whereas the knee joint rotation moment increased $(p=0.02)$. However, the knee joint abduction moment remained at the same level after CS training. SEM values were $1.6^{\circ}$ for trunk lateral lean, and $0.28 \mathrm{Nm}^{\mathrm{kg}} \mathrm{kg}^{-1}$ and 0.08 $\mathrm{Nm} \cdot \mathrm{kg}^{-1}$ for knee joint flexion and rotation moments, respectively. 
Table 3 about here

\begin{abstract}
All core endurance (prone and side planks), jump performance, agility, and core power parameters were significantly improved after CS training (Table 4).
\end{abstract}

Table 4 about here

\title{
DISCUSSION
}

The main findings of the study were as follows: 1) CS training increased trunk and pelvis rotation in the new movement direction during lateral reactive jumps; 2) lateral trunk lean was increased during lateral movements after CS training; 3) the knee joint abduction moment was not influenced by CS training; and 4) CS training improved core endurance and power, jump performance, and agility.

Perturbed lateral reactive jumps yielded significantly different segment and joint configurations. This influence of LRJ modality has already been described in the literature $(24,34)$. Moreover, during perturbed lateral reactive jumps, the trunk and pelvis positioning were influenced by CS training. Indeed, trunk lateral flexion and pelvis lateral tilt were significantly increased after CS training, and both right axial rotations of these segments were enhanced after training. Five weeks of dynamic and functional core stability training resulted in a better positioning of the core segments in the transversal plane with increased rotation towards the new movement direction during lateral reactive jumps, which is in agreement with results found by Weltin et al. (35) after a training program combining plyometric exercises with perturbation compared to plyometric training alone. However, core segments were significantly less orientated towards the new movement direction in the frontal plane. 
Although significant, these changes in trunk lateral flexion and pelvis lateral tilt were rather small given the standard error of the measurements $\left(0.7^{\circ}\right.$ and $1.3^{\circ}$, respectively). During unanticipated cutting maneuvers, the better orientation of trunk and pelvis segments in the transversal plane seen during LRJ was lacking, which differs from the results of Weltin et al. (35). In the frontal plane, pelvis medial tilt was kept at the same level and only a small $2^{\circ}$ increase in trunk lateral flexion (with respect to $1.6^{\circ} \mathrm{SEM}$ ) was observed after CS training. Core stability improvements during lateral movements after dynamic and functional core stability training were limited and, therefore, our first hypothesis was mainly rejected. Although the better trunk and pelvis orientation towards the new movement direction seen in the transversal plane during LRJ might possibly reflect a reduced knee injury risk $(6,8,10,11)$, this was lacking during unanticipated cutting maneuvers. The increased trunk lateral lean during lateral movements after CS training could be seen as a negative effect on knee joint loads $(8,18,36)$. However, in the absence of an increased knee joint abduction angle and moment, this could also reflect a strategy to move the center of mass towards the new direction during the stance phase (27). Nevertheless, training programs seeking to reduce trunk lateral lean during cutting maneuvers should instead focus on a specific technique training that is more effective (7). However, the present dynamic and functional CS training influenced core stability during lateral movements to a larger extent than previous SC programs $(17,38)$.

Despite some core stability alterations after CS training, the knee joint abduction moment was kept at the same level during unanticipated cutting maneuvers. This is in line with previous studies $(17,35,38)$ and confirms our second hypothesis. However, other consequences at the knee joint level were found during unanticipated cutting maneuvers with a lower knee flexion moment and higher knee external rotation moment. Although significant, the increased knee external rotation moment $\left(0.01\right.$ to $\left.0.08 \mathrm{Nm} \cdot \mathrm{kg}^{-1}\right)$ was small given its 
standard error of measurement and compared to the larger influence on knee joint flexion (a reduction from 0.73 to $0.26 \mathrm{Nm} \cdot \mathrm{kg}^{-1}$ ), which would probably mean an overall knee joint load reduction after CS training. During lateral reactive jumps, knee joint stability remained at the same level after CS training.

The present dynamic and functional CS training influenced all tested performance parameters as hypothesized. The significant enhanced core muscle endurance and power are in line with the literature $(17,29,33)$. Jump performance and agility were also increased by training. Therefore the present CS exercises can be implemented by athletes in their regular training to improve performance and underlines the importance of implementing dynamic movements, added resistance and functional exercises during training, as described by Hibbs et al. (15).

This study has some limitations that need to be considered. First, the lack of any control group might reduce the impact of the results found for the intervention group. However, the training was designed according to evidence from the literature based on a model for potential performance benefits (15) and a framework for ACL injury prevention (9). Moreover, the testing procedure used in the present study was comparable to another training study with two different interventions (35), which helped with the interpretation of the present findings. The sprint time during the agility test was measured manually, which might decrease accuracy of the measurement. However, according to the substantial improvement of all other performance parameters, we are confident of our conclusion about the enhanced athletic performance after core stability training. Finally, the motor learning principles behind the training procedure referred mainly to an internal focus of attention and explicit learning. Future research in this field might consider i) directing the subject's attention to the effects of the movements on the environment, ii) strengthening the implicit learning, and iii) promoting 
self-controlled learning in order to optimize the training intervention and therefore improve participants' functional performance (40).

In conclusion, a 5-week dynamic and functional core stability training program improves core muscles endurance and power improvement, as well as increasing jump performance and agility. This might explain the better orientation of the trunk and pelvis towards the new movement direction during lateral reactive jumps, especially in the transversal plane. However, this improved core stability was not observed during a more challenging and dynamic task, i.e., unanticipated cuttings, during which knee joint abduction remained at the same level after the training.

\section{PRACTICAL APPLICATIONS}

The practical application of this research will allow strength and conditioning professionals to setup training programs with relevant exercises targeting the enhancement of core stability. By using different variations of lateral shuffles, medicine ball throws, or cable lift tasks, as described in the present study, it is possible to increase core endurance and power, as well as jump performance and agility of trained individuals. These exercises can be performed in every gym with very simple sport equipment. Ultimately, these improvements are likely to enable athletes to perform at a better level with a reduced risk of knee joint injury.

\section{ACKNOWLEDGMENTS}

We would like to thank the participants for their time and commitment throughout this study. The authors report no conflict of interest and state that the results of the present study do not constitute endorsement of the product by the authors or the NSCA. 


\section{REFERENCES}

1. Agel, J, Arendt, EA, and Bershadsky, B. Anterior cruciate ligament injury in national collegiate athletic association basketball and soccer: a 13-year review. Am J Sports Med 33: 524-530, 2005.

2. Alentorn-Geli, E, Myer, GD, Silvers, HJ, et al. Prevention of non-contact anterior cruciate ligament injuries in soccer players. Part 1: mechanisms of injury and underlying risk factors. Knee Surg Sports Traumatol Arthrosc 17: 705-729, 2009.

3. Araujo, S, Cohen, D, and Hayes, L. Six weeks of core stability training improves landing kinetics among female capoeira athletes: a pilot study. J Hum Kinet 45: 27-37, 2015.

4. Chimera, NJ, Swanik, KA, Swanik, CB, and Straub, SJ. Effects of plyometric training on muscle-activation strategies and performance in female athletes. $J$ Athl Train 39: 24-31, 2004.

5. Cohen, J. A power primer. Psychol Bull 112: 155-259, 1992.

6. David, S, Komnik, I, Peters, M, Funken, J, and Potthast, W. Identification and risk estimation of movement strategies during cutting maneuvers. J Sci Med Sport, 20: 10751080, 2017.

7. Dempsey, AR, Lloyd, DG, Elliott, BC, Steele, JR, and Munro, BJ. Changing sidestep cutting technique reduces knee valgus loading. Am J Sports Med 37: 2194-2200, 2009.

8. Dempsey, AR, Lloyd, DG, Elliott, BC, et al. The effect of technique change on knee loads during sidestep cutting. Med Sci Sports Exerc 39: 1765-1773, 2007.

9. Donnelly, CJ, Elliott, BC, Ackland, TR, et al. An anterior cruciate ligament injury prevention framework: incorporating the recent evidence. Res Sports Med 20: 239-262, 2012.

10. Donnelly, CJ, Lloyd, DG, Elliott, BC, and Reinbolt, JA. Optimizing whole-body kinematics to minimize valgus knee loading during sidestepping: Implications for ACL 
injury risk. J Biomech 45: 1491-1497, 2012.

11. Frank, B, Bell, DR, Norcross, MF, et al. Trunk and hip biomechanics influence anterior cruciate loading mechanisms in physically active participants. Am J Sports Med 41: 2676-2683, 2013.

12. Hewett, TE, and Myer, GD. The mechanistic connection between the trunk, hip, knee, and anterior cruciate ligament injury. Exerc Sport Sci Rev 39: 161-166, 2011.

13. Hewett, TE, Myer, GD, Ford, KR, et al. Biomechanical measures of neuromuscular control and valgus loading of the knee predict anterior cruciate ligament injury risk in female athletes: a prospective study. Am J Sports Med 33: 492-501, 2005.

14. Hewett, TE, Torg, JS, and Boden, BP. Video analysis of trunk and knee motion during non-contact anterior cruciate ligament injury in female athletes: lateral trunk and knee abduction motion are combined components of the injury mechanism. Br J Sports Med 43: 417-422, 2009.

15. Hibbs, AE, Thompson, KG, French, D, Wrigley, A, and Spears, I. Optimizing performance by improving core stability and core strength. Sports Med 38: 995-1008, 2008

16. Hughes, G. A review of recent perspectives on biomechanical risk factors associated with anterior cruciate ligament injury. Res Sports Med 22: 193-212, 2014.

17. Jamison, ST, McNeilan, RJ, Young, GS, et al. Randommized Controlled Trial of the effects of a trunk stabilization program on trunk control and knee loading. Med Sci Sports Exerc 44: 1924-1934, 2012.

18. Jamison, ST, Pan, X, and Chaudhari, AMW. Knee moments during run-to-cut maneuvers are associated with lateral trunk positioning. J Biomech 45: 1881-1885, 2012.

19. Kibler, WB, Press, J, and Sciascia, A. The role of core stability in athletic function. Sports Med 36: 189-198, 2006. 
20. Koga, H, Nakamae, A, Shima, Y, et al. Mechanisms for noncontact anterior cruciate ligament injuries: knee joint kinematics in 10 injury situations from female team handball and basketball. Am J Sports Med 38: 2218-2225, 2010.

21. McGill, SM, Childs, A, and Liebenson, C. Endurance/time for low back stabilization exercise : clinical targets for testing and training from a normal database. Arch Phys Med Rehabil 80: 941-944, 1999.

22. Mornieux, G, Gehring, D, Fürst, P, and Gollhofer, A. Anticipatory postural adjustments during cutting manoeuvres in football and their consequences for knee injury risk. $J$ Sports Sci 32: 1255-1262, 2014.

23. Mornieux, G, Gehring, D, Tokuno, C, Gollhofer, A, and Taube, W. Changes in leg kinematics in response to unpredictability in lateral jump execution. Eur J Sport Sci 14: 678-85, 2014.

24. Mornieux, G, Weltin, E, Pauls, M, Rott, F, and Gollhofer, A. Influence of a full-body compression suit on trunk positioning and knee joint mechanics during lateral movements. J Appl Biomech 33: 261-267, 2017.

25. Myer, GD, Sugimoto, D, Thomas, S, and Hewett, TE. The influence of age on the effectiveness of neuromuscular training to reduce anterior cruciate ligament injury in female athletes. Am J Sports Med 41: 203-215, 2013.

26. Pappas, E, Nightingale, EJ, Simic, M, et al. Do exercises used in injury prevention programmes modify cutting task biomechanics? A systematic review with meta-analysis. Br J Sports Med 49: 673-680, 2015.

27. Patla, AE, Adkin, A, and Ballard, T. Online steering: coordination and control of body center of mass, head and body reorientation. Exp Brain Res 129: 629-634, 1999.

28. Pfile, KR, Hart, JM, Herman, DC, et al. Different exercise training interventions and drop-landing biomechanics in high school female athletes. J Athl Train 48: 450-462, 
2013.

29. Prieske, O, Muehlbauer, T, Borde, R, et al. Neuromuscular and athletic performance following core strength training in elite youth soccer: role of instability. Scand J Med Sci Sports 26: 48-56, 2016.

30. Renstrom, P, Ljungqvist, A, Arendt, E, et al. Non-contact ACL injuries in female athletes: an International Olympic Committee current concepts statement. Br J Sports Med 42: 394-412, 2008.

31. Stewart, PF, Turner, AN, and Miller, SC. Reliability, factorial validity, and interrelationships of five commonly used change of direction speed tests. Scand J Med Sci Sports 24: 500-506, 2014.

32. Sugimoto, D, Myer, GD, Barber Foss, KD, and Hewett, TE. Specific exercise effects of preventive neuromuscular training intervention on anterior cruciate ligament injury risk reduction in young females: meta-analysis and subgroup analysis. Br J Sports Med 49: 282-289, 2015.

33. Watson, T, Graning, J, McPherson, S, et al. Dance, balance and core muscle performance measures are improved following a 9-week core stabilization training program among competitive collegiate dancers. Int J Sports Phys Ther 12: 25-41, 2017.

34. Weltin, E, Gollhofer, A, and Mornieux, G. Effect of gender on trunk and pelvis control during lateral movements with perturbed landing. Eur J Sport Sci 16: 182-189, 2016.

35. Weltin, E, Gollhofer, A, and Mornieux, G. Effects of perturbation or plyometric training on core control and knee joint loading in women during lateral movements. Scand J Med Sci Sports 27: 299-308, 2017.

36. Weltin, E, Mornieux, G, and Gollhofer, A. Influence of gender on trunk and lower limb biomechanics during lateral movements. Res Sports Med 23: 265-277, 2015.

37. Weston, M, Hibbs, AE, Thompson, KG, and Spears, R. Isolated core training improves 
sprint performance in National-Level Junior. Int J Sports Physiol Perform, 10: 204-210, 2015.

38. Whyte, EF, Richter, C, O'Connor, S, and Moran, KA. Effects of a dynamic core stability program on the biomechanics of cutting maneuvers: a randomized controlled trial. Scand J Med Sci Sports 28: 452-462, 2018.

39. Zazulak, BT, Hewett, TE, Reeves, NP, Goldberg, B, and Cholewicki, J. Deficits in neuromuscular control of the trunk predict knee injury risk: a prospective biomechanicalepidemiologic study. Am J Sports Med 35: 1123-1130, 2007.

40. Gokeler, A, Neuhaus, D, Benjaminse, A, Grooms, DR, and Baumeister, J. Principles of motor learning to support neuroplasticity after ACL injury: implications for optimizing performance and reducing risk of second ACL injury. Sports Med Feb. 21, 2019. 
1

2

3

4

5

6

7

8

9

10

11

12

13

14

15

16

17

18

19

20

21

22

23

24

25

26

27

28

29

30

31

32

33

34

35

36

37

38

39

40

41

42

43

44

45

46

47

48

49

50

51

52

53

54

55

56

57

58

59

60

61

62

63

64

65

\section{Figure}

Figure 1 Schematic view of the cable lift test. 


\section{Tables}

Table 1 Overview of the dynamic and functional core stability program.

* 30sec break between sets and 2 min30sec between exercises.

** warm-up based on forward lunge, lateral lunge, backward lunge with a twist, drop lunge, hand walk, hip crossover and scorpion exercises.

*** resistance was induced by elastic ropes (three degrees of resistance were generated).

Table 2 Mean \pm SD values of the different kinematic variables averaged over all three lateral reactive jumps (LRJ) modalities (pooled) performed before (PRE) and after (POST) the core stability training intervention.

The influence of the core stability training intervention is reported using $p, 95 \%$ confidence interval $(95 \% \mathrm{CI})$ and effect size $(\mathrm{ES})$.

${ }^{*}, * *, * * *$ indicates significant differences between PRE and POST with $p<0.05, p<0.01$ and $p$ $<0.001$, respectively.

Table 3 Mean \pm SD values of the different kinematic and joint moment variables during cutting maneuvers performed before (PRE) and after (POST) the core stability training intervention.

The influence of the core stability training intervention is reported using $p, 95 \%$ confidence interval $(95 \% \mathrm{CI})$ and effect size $(\mathrm{ES})$.

**, ${ }^{* * *}$ indicates significant differences between PRE and POST with $p<0.01$ and $p<0.001$, respectively. 
Table 4 Mean \pm SD values of the different core endurance and athletic performance variables before (PRE) and after (POST) the core ctability training intervention.

The influence of the core stability training intervention is reported using $p, 95 \%$ confidence interval $(95 \% \mathrm{CI})$ and effect size (ES).

${ }^{*}, * *,{ }^{* * *}$ indicates significant differences between PRE and POST with $p<0.05, p<0.01$ and $p$ $<0.001$, respectively. 

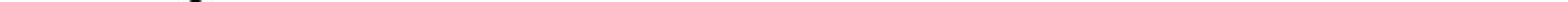

Figure 1

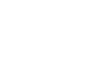


Warm-up (5min)**

$1-3$

Medicine ball throw : right technique learning with a $2 \mathrm{~kg}$ ball $(5 \mathrm{~min})$

Medicine ball throw ( $2 \mathrm{~kg}$ ball): $3 \times 10$ on each side $(5 \mathrm{~min})$

Cable lift: right technique learning with the first degree of resistance $(5 \mathrm{~min})^{* * *}$

Warm-up (5min)

Medicine ball throw ( $2 \mathrm{~kg}$ ball): $3 \times 10$ on each side $(5 \mathrm{~min})$

$4-6$

Cable lift (first degree of resistance): $3 \times 10$ on each side (5min)

Lateral shuffle: right technique learning $(5 \mathrm{~min})$

Warm-up (5min)

Medicine ball throw ( $3 \mathrm{~kg}$ ball): $3 \times 10$ on each side $(5 \mathrm{~min})$

$7-9$

Cable lift (second degree of resistance): 3 x 10 on each side ( $5 \mathrm{~min}$ )

Lateral shuffle: mirror drills with partner (5min)

Warm-up (5min)

Medicine ball throw ( $3 \mathrm{~kg}$ ball): $3 \times 10$ on each side $(5 \mathrm{~min})$

$10-12 \quad$ Cable lift (third degree of resistance): $3 \times 10$ on each side (5min)

Lateral shuffle: shuffles with increased resistance through elastic ropes

attached to the pelvis $(5 \mathrm{~min})$

Warm-up (5min)

Cable lift (third degree of resistance): $3 \times 10$ on each side ( $5 \mathrm{~min}$ )

Lateral shuffle: shuffles with increased resistance through elastic ropes

$13-15$

attached to the pelvis $(5 \mathrm{~min})$

Lateral shuffle: shuffles with perturbation through elastic ropes more or less

released by the athletic coach ( $5 \mathrm{~min})$ 


\begin{tabular}{|c|c|c|c|c|c|}
\hline Variable & PRE (pooled LRJ) & POST (pooled LRJ) & $p$ value & $95 \% \mathrm{CI}$ & ES \\
\hline Trunk Forward Lean $\left({ }^{\circ}\right)$ & $27.2 \pm 5.1$ & $29.1 \pm 5.0$ & 0.06 & {$[-3.9,0.1]$} & 0.47 \\
\hline Trunk Lateral Lean $\left(^{\circ}\right)$ & $-5.6 \pm 4.7$ & $-7.0 \pm 5.0^{*}$ & 0.04 & {$[0.1,2.9]$} & 0.50 \\
\hline Trunk Axial Rotation $\left(^{\circ}\right)$ & $2.3 \pm 9.1$ & $6.3 \pm 10.2 * *$ & 0.002 & {$[-5.2,-1.5]$} & 1.1 \\
\hline Pelvis Forward Tilt $\left(^{\circ}\right)$ & $17.7 \pm 6.4$ & $18.4 \pm 4.6$ & 0.35 & {$[-4.7,0.5]$} & 0.27 \\
\hline Pelvis Medial Tilt $\left(^{\circ}\right)$ & $-5.6 \pm 4.9$ & $-7.6 \pm 4.2^{* *}$ & 0.002 & {$[0.9,3.2]$} & 0.77 \\
\hline Pelvis Axial Rotation $\left(^{\circ}\right)$ & $4.9 \pm 8.9$ & $8.6 \pm 7.4 * * *$ & $<0.001$ & {$[-5.4,-2.2]$} & 1.0 \\
\hline Knee Flexion $\left(^{\circ}\right)$ & $58.8 \pm 5.6$ & $57.6 \pm 6.6$ & 0.06 & {$[-0.1,2.5]$} & 0.54 \\
\hline Knee Abduction $\left(^{\circ}\right)$ & $7.1 \pm 5.2$ & $8.3 \pm 3.8$ & 0.25 & {$[-3.5,1.0]$} & 0.23 \\
\hline Knee Ext. Rotation $\left(^{\circ}\right)$ & $-10.4 \pm 7.1$ & $-10.3 \pm 5.2$ & 0.97 & {$[-2.6,2.5]$} & 0.02 \\
\hline
\end{tabular}




\begin{tabular}{|c|c|c|c|c|c|}
\hline Variable & PRE & POST & $p$ value & $95 \% \mathrm{CI}$ & $\mathrm{ES}$ \\
\hline Trunk Forward Lean $\left(^{\circ}\right)$ & $7.5 \pm 5.2$ & $6.0 \pm 5.7$ & 0.28 & {$[-1.4,4.4]$} & 0.26 \\
\hline Trunk Lateral Lean $\left(^{\circ}\right)$ & $-3.8 \pm 3.9$ & $-5.8 \pm 4.4^{*}$ & 0.03 & {$[0.3,3.3]$} & 0.70 \\
\hline Trunk Axial Rotation $\left(^{\circ}\right)$ & $-9.0 \pm 8.6$ & $-8.1 \pm 7.7$ & 0.59 & {$[-4.6,2.7]$} & 0.11 \\
\hline Pelvis Forward Tilt $\left(^{\circ}\right)$ & $17.5 \pm 5.7$ & $17.6 \pm 4.6$ & 0.90 & {$[-1.5,1.2]$} & 0.03 \\
\hline Pelvis Medial Tilt $\left({ }^{\circ}\right)$ & $13.0 \pm 4.7$ & $11.8 \pm 3.8$ & 0.29 & {$[-1.1,3.5]$} & 0.23 \\
\hline Pelvis Axial Rotation $\left(^{\circ}\right)$ & $7.7 \pm 6.9$ & $9.9 \pm 7.3$ & 0.15 & {$[-5.3,0.9]$} & 0.35 \\
\hline Knee Flexion Moment $\left(\mathrm{Nm} \cdot \mathrm{kg}^{-1}\right)$ & $0.73 \pm 0.71$ & $0.26 \pm 0.80 * *$ & 0.01 & {$[0.11,0.81]$} & 0.69 \\
\hline Knee Abduction Moment (Nm. $\left.\mathrm{kg}^{-1}\right)$ & $0.74 \pm 0.45$ & $0.71 \pm 0.48$ & 0.88 & {$[-0.27,0.31]$} & 0.05 \\
\hline Knee Ext. Rotation Moment $\left(\mathrm{Nm} \cdot \mathrm{kg}^{-1}\right)$ & $0.01 \pm 0.10$ & $0.08 \pm 0.12 *$ & 0.02 & {$[-0.13,-0.02]$} & 0.64 \\
\hline
\end{tabular}




\begin{tabular}{lccccc}
\hline Variable & PRE & POST & P value & $95 \%$ CI & ES \\
\hline Prone Plank (s) & $79.0 \pm 22.1$ & $93.3 \pm 26.7 * *$ & 0.006 & {$[-23.9,-4.8]$} & 0.81 \\
Side Plank (s) & $51.3 \pm 17.2$ & $69.9 \pm 19.4^{* * *}$ & $<0.001$ & {$[-25.5,-11.6]$} & 1.4 \\
Broad Jump (m) & $1.78 \pm 0.19$ & $1.86 \pm 0.18^{* * *}$ & $<0.001$ & {$[-12.6,-4.8]$} & 0.99 \\
Side Jump (m) & $1.71 \pm 0.16$ & $1.78 \pm 0.13^{* *}$ & 0.002 & {$[-10.8,-2.7]$} & 0.80 \\
Agility (s) & $5.4 \pm 0.3$ & $5.2 \pm 0.3^{* * *}$ & $<0.001$ & {$[0.1,0.3]$} & 0.98 \\
Core Power (W) & $265 \pm 56$ & $300 \pm 43^{* * *}$ & $<0.001$ & {$[-49,-19]$} & 1.2 \\
\hline
\end{tabular}

